\title{
Heat design of optoelectronic devices in training optics specialists
}

\section{G. Dulnev, Elena Doulneva}

G. N. Dulnev, Elena G. Doulneva, "Heat design of optoelectronic devices in training optics specialists," Proc. SPIE 1603, Education in Optics, (1 March 1992); doi: 10.1117/12.57891

SPIE. Event: Education in Optics, 1991, Leningrad, Russian Federation 


\section{HEAT DESIGN OF OPTO-ELECTRONICAL DEVICES IN TRAINING OPTICS SPECIALISTS.

\author{
G.N.Doulnev, E.G.Doulneva
}

St.Petersburg Institute of Fine Mechanics and Optics 14, Sablinskaya st., St.Petersburg 197101 Russia

The use of a hierarchial principle in assemblying opto-electronical devices. Opto-electronical devices (herein after referred to as OED) are complex systems, whose components are optical and mechanical groups, receivers and sources of radiation, electronic blocks, etc. The OED temperature rate stipulates both the quality and reliability of its groups and blocks as well as functioning the device as a whole. Therefore analysing temperature fields and their impact on heat aberrations is part of the whole process in projecting a device, and also in educating future optics designers.

Basic OED schemes and designs are rather sufficientiy described in technical literature so that we can emphasize the following levels of design work [1].

The first level is represented by elements, that is parts of a whole design, which are inseparable in a certain integral design, e.g. - parts of an optical system (mirrors, lenses, prisms, blemds, etc.), parts of mechanical groups (scanning and monitor systems drives, various transformers, modulators), sources of radiation (lasers, gas-discharge, lamps, light-conductor elements), etc. Fig.1, a shows certain elements of an OED : mirrors - 1 and 2 , blend - 3 ).

The second level is represented by separate design blocks, made up of first level elements. Normally they have their own body and in some cases - their own heat rate ensurance system. For example: kryostates, connected with cooler blocks through heat-exchangers, heat bridges or heat tubes, thermoelectric battaries, etc. Fig.1,b shows an OED object-glass, consisting of the main and secondary compensatory lenses $-3-6$ and a blend -7 . 


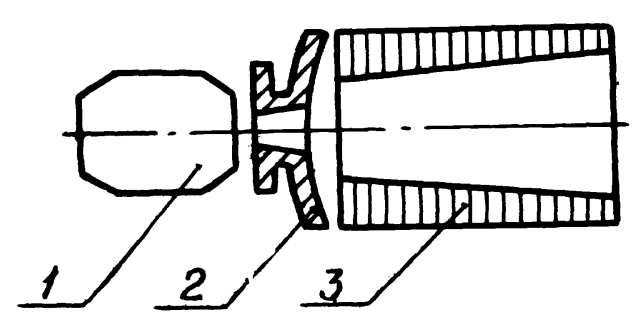

a
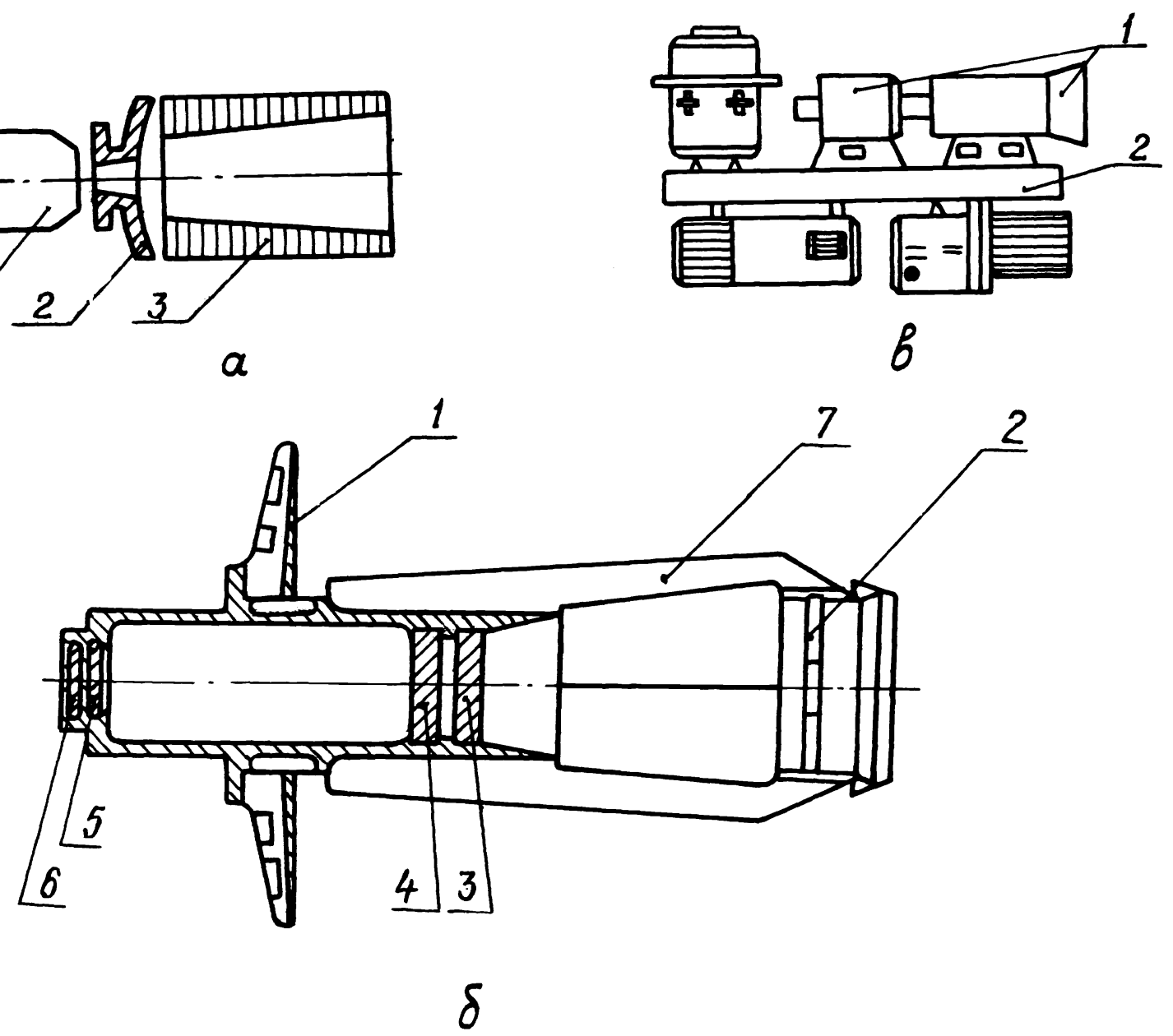

Fig. 1 .

The third design level is represented by an opto-electronical device, made up of separate second level blocks and first level elements, which have a common cooler system. Cooler system use kryostates, thermobatteries, various cooling appliancies and radiators; heating devices are used as heaters.

The fourth level is represented by a group of devices and appliancies 1, placed in one device section or on a separate platform 2 (fig.1,d).

A step-by-step approach to modelling opto-electronical devices. 
The general OED heat model is a system with a quite a number of irregulary shaped areas, randomly situated in space and having their heat sources and heat streams.

Temperature fields of such a system can be best described as a system of differential equations for solid-state heat conductivity, as well as that of movement, inseparability and state of cooler agents streams under appropriate marginal and starting conditions.

A mathematical realization for such a system calls for numerical methods and modern computers. Computer processing may be considerably simplified if a step by step approach to modelling (as described in $[4,5]$ ) is used.

The process of enlargement (composition) and detailing (decomposition), which form part of the step by step procedure should be subjected to the hierarchy, adopted in the given design. Mathematical models of OED heat rate correspond to different levels of the designer's hierarchy and make possible the following calculations: average temperatures of devices and appliancies, placed in one section or on one platform; average temperatures of certain devices and groups (optical, electronical, mechanical, etc.), making up one OED; average temperatures of separate design elements (lenses, mirrors, bodies, etc.); temperature fields of separate elements of a certain device.

Let us analyse the first step of the calculation, which permits to determine the average surface temperatures of devices in a given section. Devices and mechanisms exchange heat among themselves with machine-tools or with the surface of the platform as well as with the environment. This kind of heat exchange is effected by conduction, radiation and convection in atmosphere, and also by radiation and conduction in outer space. Devices themselves are heat sources, producing heat radiation, plus external heat sources such as solar radiation. At this stage we shall consider temperature fields of separate devices and 
mechanisms to be equal. Then the $P_{2}$ heat stream in the $i$-area goes to increasing of enthalpy $c_{j} * d T_{j} / d \tau$ at this area and is transferred to other bodies $j$, that is $\Sigma\left(T_{i}-T_{j}\right) \sigma_{i j}$ and to the $\left(T_{i}-T_{j}\right) \sigma_{i c}$ area, and is also channeled through a heat tube, thermobattery or a cooler device $Q_{i}$ :

$C_{i} * d T_{i} / d \tau+\sum_{j=1}^{N}\left(T_{i}-T_{j}\right) \sigma_{i j}=P_{i}-Q_{i}-\left(T_{i}-T_{C}\right) \sigma_{i c} ;$

$T_{c}=$ const $, i=1,2, \ldots, N$

under primary conditions $T_{i}=T_{i 0}$ when $\tau=0$.

Formulae for calculating heat conductivity $\sigma$ and heat stream $Q_{2}$, channeled out of the device through a heat tube, by a thermobattery, a cooler machine, are presented in [5]. Subsequent stages of our calculation, corresponding to the appropriate mathematical models, and ways of their realization are analysed in [5].

Designing a thermostable space telescope. Let us analyse some pecularities of designing a thermostable telescope (herein after referred to as "an object-glass") using a television camera which our "Vega" space probe was equipped with.

The TV narrow-field-of-view camera was intended to obtain black and white and spectrozonal images of the Galley comet's core, as well as to monitor the turning platform which was used to focus scientific instruments on the comet's core. The TV camera functions in outer space. Low temperature and high density of the solar radiation may cause considerable unevennes of the optical system's temperature field, as a result of which thermooptical aberrations may occur (primarily - it may fall out of focus). Therefore in designing a telescope for the "Vega" TV system conditions must be created under which thermooptical aberration parameters will not exceed the acceptable limit. 


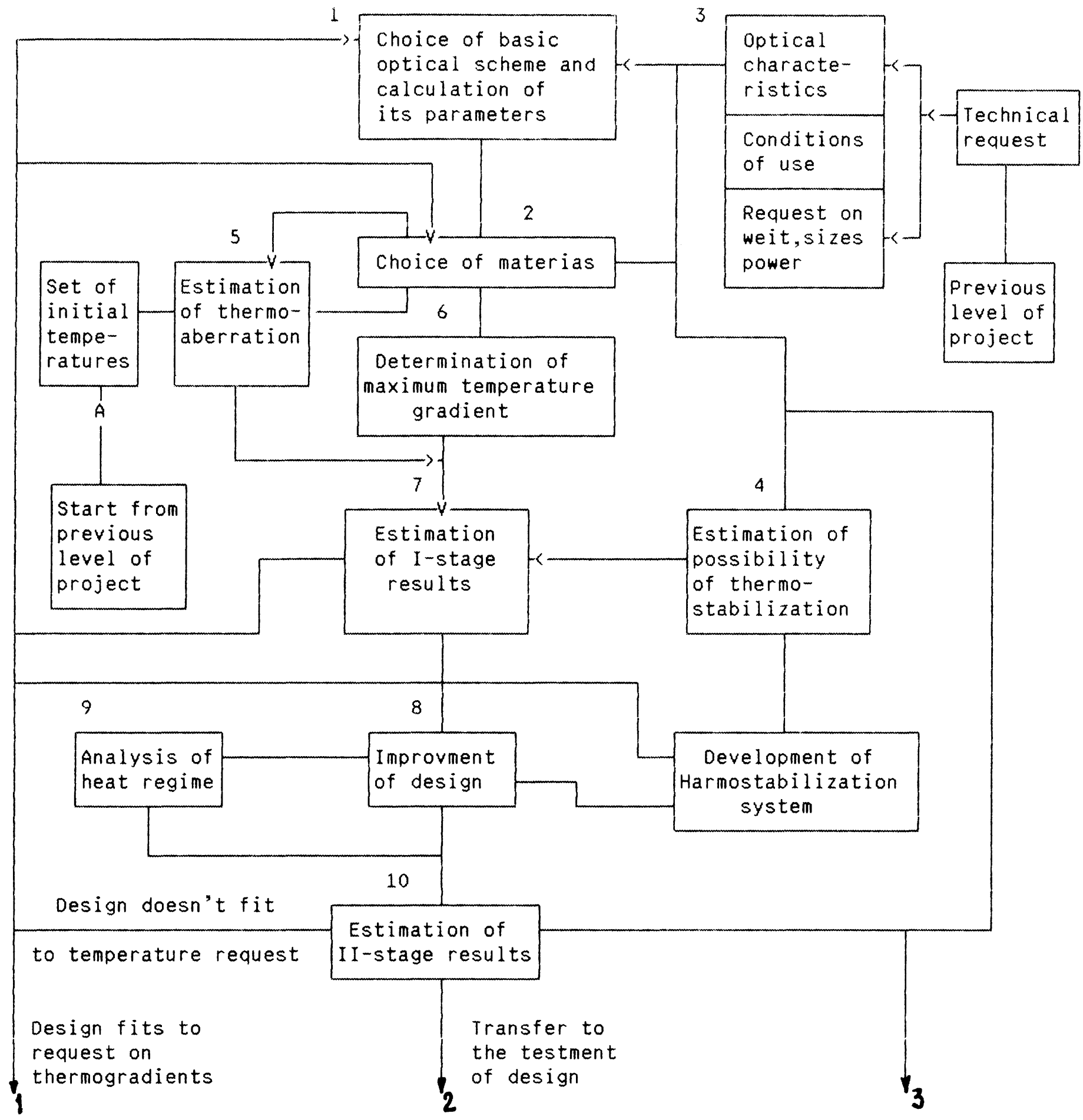

Fig.2 (see also next page). 


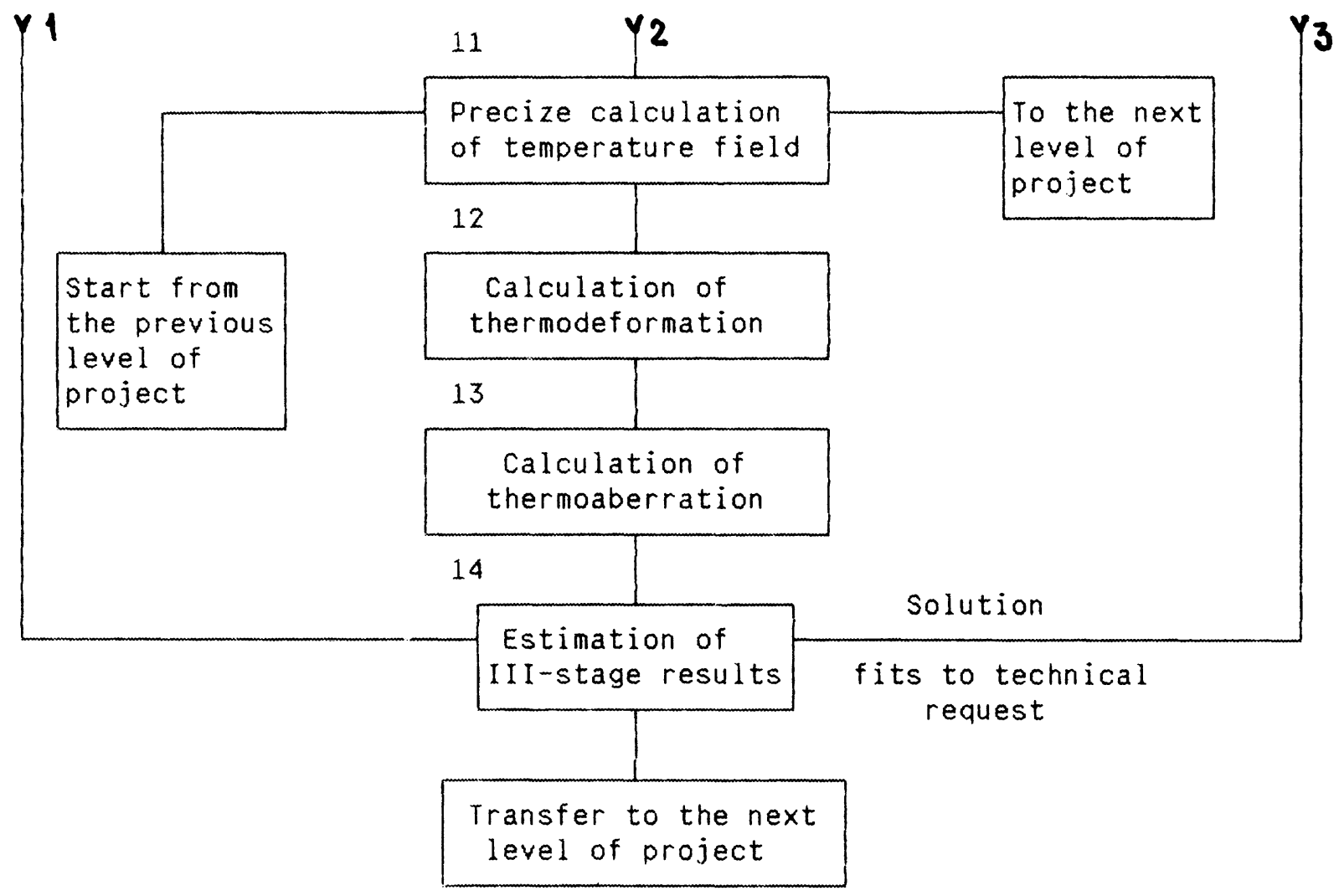

Fig. 2 .

To solve this problem an analysis of the device's heat and optical parameters was made, which made it possible to come over to the next task - that of synthezing the design, in other words - creating a thermostable optical system.

The synthesis procedure was made by a step by step method. At the first stage the basic optical scheme optical and design elements were adopted, acceptable temperature fluctuations were considered (see block-scheme, fig.2).

The choice of the basic optical scheme, which will determine subsequent design work. Optical solutions classification, an approach to choosing the optimal scheme and the formalization of thr procedure is borrowed from [6]. Fig.2 presents a block-scheme of the first period of designing, where block 1 opens up the 
procedure with the choice of the basic scheme and calculation of the optical system (OS) parameters, block 3 including initial conditions for solving the task, that is: a technical order (TO) for optical parameters and quality of the picture, operation requirements, limitations for the weight and dimensions of the device, ways of active thermostating (block 4 ). Let us note that as far as the "Vega" TV cameras are concerned active thermostating was out of the question because of strict requirements to the device's mass and its energy consumption.

Fig.1,b shows the chosen basic optical scheme. After the optical scheme parameters had been calculated, material for making mirrors, lense frames, object-glass body and block of detectors was chosen. It was taken into account that thermooptical aberrations are determined by three factors: changes in the general level of the optical system temperature, temperature fluctuations between the optical elements and temperature gradient in the elements. This considered, various ways of solving the task can be used. One of them is the choice of materials with extremely low linear enlarging coefficient. In this case mirrors must be of quartz, pyrex or sitall whereas the elements of the design should be made of invar.

Another way is to make mirrors, the elements of design using one and the same material, e.g. titanium. Temperature fluctuations in the optical system and temperature gradients will be lower than in the previous case (because of the material's high conductivity), the object-glass mass also being considerably smaller. The choice of the way is to a greater extent determined by the manufacture opportunities. In this case we have chosen the second way.

In order to account for such choice at this stage one should evaluate the thermoaberrations (see block 5, fig.2). This was done for two values of the even temperature field, equal to the assumed extreme values of the platform temperature: $+40^{\circ} \mathrm{C}$ and $-20^{\circ} \mathrm{C}$ (see [7]). It is shown that the scattering area diameter $d$, 
as a result of falling out of focus is caused by temperature fluctuatuions $\Delta T_{12}$ between mirrors 1 and 2 , 1 inear enlarging coefficient of the material $\alpha$, diameter $D$ of the main mirror by dependence:

$d=1.125 \mathrm{D} \propto \Delta T_{12}$.

Putting the values $D=240 \mathrm{~mm}, \alpha=8.5 * 10^{-6} \mathrm{~K}^{-1}$ (titanium), and chosing the minimum size for the radiation receiver element $\mathrm{d}<0.02 \mathrm{~mm}$ (CCD-matrix element) we shall have $\Delta T<8 \mathrm{~K}$. Thus we have answered the question about the conditions in which the device functions, the limits of acceptable thermoaberrations and temperature fluctuations among mirrors. This makes it possible to procede with the second stage of designing - projecting a telescope (block 8, fig.3) with acceptable temperature levels of the optical system (block 9). The analysis shows that the TV camera heat rate $(T)$ is basically determined by heat exchange with outer space $(K)$ as well as with the blend ( $B$ ) and the platform $(P)$. Heat conductivity among these elements we shall mark $\sigma_{T K}$, $\sigma_{T B}$, $\sigma_{T P}$. For stationary conditions with the absense of heat tubes and other devices $Q=0$ the equation (1) provides:

$T_{T}=\left(T_{P} \sigma_{T K}+T_{B} \sigma_{T B}+T_{T P} \sigma_{T P}\right) /\left(\sigma_{T K}+\sigma_{T B}+\sigma_{T P}\right)$

As shown by the analysis $T_{T}$ is determined by a correlation among three conductivities, $\sigma_{T K}$ 's influence being smal1 and a 11 attention should be paid to diminishing heat connection between the TV camera and the blend ( $f i g .1, b)$, that is to "separate" them in the design and, vice versa, enhance $\sigma_{T P}$, that is the heat connection between the TV camera and the platform [7].

At the third stage of designing temperature fields parameters were specified (block 11), those of thermodeformations (block 12) and thermoaberrations (block 13), which makes it possible to judge about whether the design of the TV camera meets the technical order requirements (block 14). A more detailed analysis of these stages of designing is given in [7]. In the book you 
will find examples of designing other opto-electronical devices including pump chambers for solid-state lasers. Thermoaberrations have been taken into account caused by: temperature depended changes of refraction index, photoelasticity effect, distortion of active element of laser, which occurs because uneven heat enlarging, thermal anysithropy.

So heat processes have a considerable effect on the quality of OED functioning. They are part and parcel of designing procedures and must be taken into account in educating optics engineers. 